\title{
A Nonsense Mutation in VHL Gene Causing Von Hippel-lindau Syndrome in a Large Chinese Family
}

\author{
Xinghuan Ding \\ Department of Neurosurgery, Beijing Ditan Hospital, Capital Medical University \\ Tingyu Liang \\ Department of Neurosurgery, Beijing Ditan Hospital, Capital Medical University \\ Ben Li \\ Department of Neurosurgery, Weifang Yidu Central Hospital \\ Bo Liang \\ Department of Neurosurgery, Beijing Ditan Hospital, Capital Medical University \\ Peiliang Li \\ Department of Neurosurgery, Beijing Ditan Hospital, Capital Medical University \\ Shichao Chen \\ Department of Neurosurgery, Beijing Ditan Hospital, Capital Medical University \\ Jingjing Li \\ Department of Radiology, Beijing Ditan Hospital, Capital Medical University \\ Fang Wang \\ Department of Neurosurgery, Beijing Ditan Hospital, Capital Medical University \\ Shanjun Wang \\ Department of Neurosurgery, Weifang Yidu Central Hospital \\ Yang Cao \\ Department of Neurosurgery, Beijing Ditan Hospital, Capital Medical University \\ Jidian Sun \\ Department of Neurosurgery, Beijing Ditan Hospital, Capital Medical University \\ Xinmei Zheng \\ Department of Neurosurgery, Beijing Ditan Hospital, Capital Medical University \\ Xiaoyong Wang \\ Department of Neurosurgery, Beijing Ditan Hospital, Capital Medical University \\ Tongxin Wang \\ Department of Neurosurgery, Weifang Yidu Central Hospital \\ Enshan Feng ( $\square$ enshanfeng@126.com ) \\ Department of Neurosurgery, Beijing Ditan Hospital, Capital Medical University
}

\section{Research}

Keywords: Von Hippel-Lindau, VHL, genetics, nonsense, mutation

Posted Date: October 23rd, 2020

DOl: https://doi.org/10.21203/rs.3.rs-95115/v1

License: @ (i) This work is licensed under a Creative Commons Attribution 4.0 International License. Read Full License 


\section{Abstract}

Background

Von Hippel-Lindau (VHL) syndrome is a multi-organ neoplastic disease characterized by highly vascular and cystic tumors in central nervous system (CNS), retina and visceral lesions, which is mainly caused by germline mutations in the $V H L$ gene.

\section{Methods}

Here, a large consanguineous four-generation family with variant phenotypes of VHL disease was recruited and molecular genetics tested via Sanger sequencing.

Result

Genetic investigation detected a c.351G>A nonsense mutation in the VHL gene, that altered the reading frame downstream and created a premature TGA stop signal, resulting in severely truncated pVHL (p.Trp117Ter). This mutation is absent from public databases, and the functional prediction bioinformatic tools demonstrated that the residue is conserved and the variant is highly likely to be deleterious.

Conclution

The c.315G>A nonsense mutation in $V H L$ gene is the causal mutation of this kindred that may lead to clear familial aggregation of $\mathrm{VHL}$ disease because of the dysfunction of truncated pVHL.

\section{Background}

VHL syndrome is a hereditary, autosomal dominant (AD), multi-organ, neoplastic disease, which is caused by genetic aberrations of the tumor suppressor gene $V H L$. It is characterized by highly vascular and cystic tumors, including hemangioblastomas (HGBs) of CNS and retina, and visceral lesions such as clear-cell renal cell carcinomas (ccRCCs) and renal cysts (RC), phaeochromocytomas (PCCs), pancreatic cysts and tumors (PCTs), and papillary cystadenomas in epididymis and broad ligament [1]. CNS hemangioblastomas are the most emblematic lesion of VHL disease, occurring in up to $80 \%$ of VHL patients, mainly in the cerebellum [2]. Along with RCCs, the two manifestations present as the major causes of mortality [3]. Retinal angioma is also the common presenting feature of VHL disease affecting multiple and bilateral fundus retinal vessel in about one half of cases [4]. VHL germline mutations affect 1 in 36,000 live births with an AD fashion, and its penetrance is estimated to be more than $90 \%$ by the age of 65 years old [5]. The onset of VHL disease occurs at a mean age of 26 years [6]. Approximately $80 \%$ of VHL patients have a multigenerational family history of the disease, and the remaining cases may due to de novo or somatic mutations of the $V H L$ gene [5].

The VHL (OMIM: 608537) tumor suppressor gene, which is located on chromosome 3p25.3, consists of three exons, encoding two isoforms of VHL proteins: $\mathrm{pVHL}_{30}$ (30 kDa in length, 213 amino acids) and $\mathrm{pVHL}_{19}$ (19 kDa in length, 160 amino acids) which lacks the first 53 residues, due to alternative translation initiation site in the open reading frame of codon 54 . Both the p30 and p19 isoforms have equivalent tumor suppressor effects, and both can regulate hypoxia-inducible factor- $\mathrm{a}(\mathrm{HIFa})[7,8]$. The $\mathrm{pVHL}$ consists of two tightly coupled domains, $a$ and $\beta$, together with elongation factors $C$ and $B$ (Elongin $C$ and Elongin B), Cullin 2 (CUL2) and the RING finger protein RBX1, forming the VCB-CR complex which is crucial for pVHL function. Then prolyl-hydroxylated HIFa is recognized and binded by the VCB-CR E3 ubiquitin ligase complex and targeted for ubiquitylation (Ub) and proteolytic degradation $[8,9]$. By contrast, pVHL harbouring mutations that disrupt the complex construction is unstable and rapidly degraded by the proteasome, resulting in HIFa accumulation and then the upregulation of HIF target genes (e.g., VEGF, PDGF $\beta$, TGF a, CyclinD1, EPO etc.) involved in various processes, such as angiogenesis, proliferation, metabolism and apoptosis. The aberrant overexpression of downstream target genes thereby contribute directly to tumorigenesis. Many molecular genomic analysis studies on VHL disease have been reported, and about 585 different germline mutations within the whole coding sequence of $V H L$ have been documented (http://www.umd.be/VHL/W_VHL). In non-mosaic patients with classical $\mathrm{VHL}$ disease germline mutations detection is almost up to $100 \%$ [10]. Approximately $11 \%$ are partial or complete deletions (ranging from 0.5 to $250 \mathrm{~kb}$ ) that remove one or more $V H L$ exons, while the remaining mutations fall into two groups, missense (52\%) or small in-frame indel (6\%) substitutions and mutations predicted to cause truncated protein (nonsense $11 \%$, frameshift 13\%, splice site $7 \%$ ) [7]. And some researches have investigated that $V H L$ mutation types, mutation regions and specific mutation codons can manifest different phenotypes of VHL disease [11].

In this study, we examined a large Chinese VHL family with 17 immediate members across four generations that includes 9 VHL patients and 1 mutation carrier. Correlated genetic findings, pathogenic mechanism, clinical characteristics and the genotype-phenotype connections are discussed.

\section{Materials And Methods}

\section{Family Recruitment}

We enrolled a family of Chinese Han ethnicity (Figure 1) with 9 members diagnosed with VHL syndrome. Participants were under detailed clinical manifestations questionnaire, physical examination, necessary imaging examination (MRI of the brain and spine, abdominal ultrasonography/CT and funduscopy) and pedigree investigation by clinicians. Two $\mathrm{mL}$ of peripheral blood samples were obtained from each available family members after the 
informed written consents for experimentation with human subjects in this genetic study were obtained from all of the participants. This research was approved by the ethics committee of Beijing Ditan Hospital.

\section{Genomic DNA Preparation and Sanger sequencing}

Genomic DNA for each individual was extracted from peripheral blood lymphocytes via standard phenol-chloroform method. After the genomic DNA was isolated, the $V H L$ gene sequence was amplified by polymerase chain reaction (PCR) with the primers designed with Primer3 online software (http://primer3.ut.ee/) summarized in Table 1. Then the products, purified with Axygen-AP-GX-50 Toolkit, were sequenced from both the DNA strands of the entire coding region and the junction regions on ABI Prism 3730 Avant DNA sequencer (Applied Biosystems). After that, the reads of the sequencing was compared to a reference sequence of GRCh38 human genome.

\section{Variant-detection and Pathogenicity prediction}

Sequence analysis was performed in software SnapGene viewer (version 2.8.3) for mutation exploring. UGENE (version 35.0) was used to investigate whether the identified amino acid substitution of pVHL was conserved between different species. Computational prediction tools (PROVEAN, Gerp++, PhyloP, PhastCons, Polyphen-2, MutationTaster, CADD, ClinGen Haploinsufficiency and ExAC pLI) were used to predict the conservation and pathogenicity of the detected variant and investigate the effect of the mutated amino acid on the protein's structure and function.

And the variant was compared against publicly available databases such as the 1000 Genomes Project (http:// internationalgenome.org/), the Exome variant server, NHLBI GO Exome Sequencing Project (ESP, http://evs.gs.washington.edu/EVS/), the Exome Aggregation Consortium database (ExAC, http://exac.broadinstitute.org/), Genome Aggregation Database (gnomAD, http://gnomad.broadinstitute.org/) and the Single Nucleotide Polymorphism Database (dbSNP, https://ncbi.nlm.nih.gov/SNP/) to investigate the frequency of the variant in healthy populations.

\section{Modeling the three dimensional structure of pVHL}

IBS (version 1.0.3) were used to present the location of the variant in both the genome and protein level. The coordinates of the HIF-1a-pVHL-ElonginBElonginC complex were obtained from the RCSB protein data bank (PDB ID:1LM8). PyMol Viewer (version 1.7.4) were used to visualize the three dimensional protein model and map the mutated amino acid point onto the three dimensional model.

\section{Results}

\section{Clinical Information}

The proband ( $₫-3)$ was a 23-years-old boy presented with one week history of mild diplopia, but with no obvious positive signs of the nervous system physical examination. Magnetic resonance imaging (MRI) of the brain revealed an enhancing lesion in the left cerebellum (Figure 2A-C). Axial T1 postcontrast images demonstrate an enhancing mural nodule (red arrowhead) with a large peritumoral cyst (red arrow) in the left cerebellar hemisphere exerting a mass effect upon the midline and fourth ventricle. Abdominal computed tomography (CT) scan revealed a cyst (red arrow) in the pancreatic head (Figure 2D). On fundus evaluation (Figure 2E), there is an atypical angiomas (white arrowhead) on his left eye retina and the optic disc presents edematous (black arrow) probably because of the intracranial hypertension leading to the clinical manifestation of diplopia. A diagnosis of HGB was comfirmed by postoperative pathology of the cerebellum lesion. Because the patient had a family history of HGB, his father (III-3, Figure 2F-J) uncle (III8) aunt (III-1) grandpa (II-1) and grandma (II-5), the entire family members were examined for VHL disease. Unfortunately, multiple enhancing nodules were revealed by brain MRI (Figure 2K-M) in the proband's sister's cerebellum (ख-4), and demonstrated to be HGBs after surgical excision of the biggest one near the brainstem (Figure 2N black arrowhead) and the other three surface lesions followed by histopathological analysis of the biopsy tissues. She also have bilateral involved retinal hemangioblastomas on fundoscopic view (Figure 20-Q), which have easily recognizable globular reddish appearance with dilated feeding arteries. The proband's cousin ( $\mathbb{-}-2)$ was also diagnosed with HGB located in his cerebellum near the brainstem (Figure 2R-T). A further two affected members (II-1 and II-5) of the family had previously died from this syndrome before we got their blood. The proband's great grandfather (I-1) suffered a sudden death in his 60 s presenting with an episodic headache for 10 years suggesting the CNS HGB diagnosis. All findings consistent with Mendelian expectation for AD VHL disease trait in this family. The pedigree of the family is presented in Figure 1 and the clinical characteristics of the proband and his family members are indicated in Table 2.

\section{Mutation detection}

All the family members were further sequenced for $V H L$ gene. A nonsense heterozygous variant NM_000551 c.351G>A (p.Trp117Ter) in exon 2 of the $V H L$ gene was revealed to be present in the proband $₫-3$ (Figure $3 A$ ). And the target sequencing of this variant in the other family members revealed 6

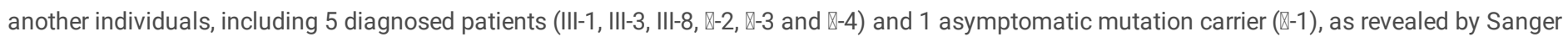
sequencing (Figure S1).

\section{Pathogenicity prediction and bioinformatics analysis}

Alignment of $\mathrm{VHL}$ amino acid sequences in numerous species revealed that the tryptophane at the 117th amino acid site and the downstream residues is evolutionarily conserved (Figure $3 \mathrm{~B}$ ), indicating that its evolution may have preserved function. In addition, this nonsense mutation is absent from public databases (1000genome, ExAC, gnomAD, ESP5400 and dbSNP), and the functional prediction bioinformatics tools (PROVEAN, Gerp++, PhyloP, 
PhastCons, Polyphen-2, MutationTaster, CADD, ClinGen Haploinsufficiency Score and ExAC pLI score) demonstrated that the residue is robustly conserved and the variant is highly likely to be deleterious (Table 3). MutationTaster predicted that c.351G>A of $V H L$ gene was a disease-causing mutation through three possible pathogenic mechanisms: Nonsense-Mediated mRNA Decay (NMD), splicing abnormality, and known disease mutation at this position. According to the prediction from the bioinformatics tools, it was indicated that the integrity and expression level of $V H L$ protein might be affected by c.351G>A.

The three dimensional protein model of HIF-1a-pVHL-ElonginB-ElonginC complex (Figure 4) was built via molecular modeling software. Ribbon diagram shows that HIF-1a binds directly to pVHL $\beta$ domain, made by the $\mathrm{N}$ segment, in particular by Hyp ${ }^{564}$ of the HIF-1a. Meanwhile, the mutated amino acid point of $\mathrm{W} 117$ is spatially close to Hyp ${ }^{564}$ (at least $3.5 \mathrm{~A}$ ).

\section{Discussion}

Clinical diagnostic criteria introduced in 1964 [12] enabled the diagnosis of VHL disease in sporadic patients who had two manifestations (such as two HGBs or a HGB and a visceral tumor), and in patients who had only a single simple manifestation (a CNS HGB or a visceral lesion) but with family history of VHL disease. Molecular genetic testing for early identification of the patients improves diagnostic certainty and erases the psychological burden of at-risk family members who have not inherited the pathogenic variant. In the present study, using Sanger sequencing, we successfully identified a novel nonsense variant, c.351G>A (p.Trp117Ter), in the second exon of VHL, which was heterozygous in 6 VHL-diagnosed members (III-1, III-

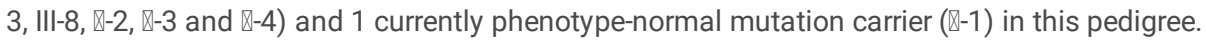

From the bioinformatics analysis, we found that the c.351G>A variant is absent from public databases, and predicted to be deleterious by bioinformatics tools. The residue p.Trp117 in pVHL which is located within the $\beta$-domain (Figure $3 \mathrm{C}$ ) and maps to hydrophobic core residue important for the structural integrity of the $\beta$ sandwich [13], is evolutionarily conserved, suggesting that this amino acid is important for maintaining the protein's structure and function.

pVHL contains two tightly coupled functional domains, the a-domain and the $\beta$-domain, held together by two short polypeptide linkers (residues 154 to 156 and 189 to 194) and a polar interface that is stabilized by hydrogen-bond networks from the $\mathrm{H} 1$ helix, the $\beta$ sandwich, and Elongin $\mathrm{C}$ [13]. The adomain is responsible for directly binding to Elongin $\mathrm{C}$, which consists of three a-helices $(\mathrm{H} 1, \mathrm{H} 2$ and $\mathrm{H} 3)$ located at amino acid residues 155-192, whereas the $\beta$-domain is the substrate recognition region of pVHL, which contains seven-stranded $\beta$ sandwiches (residues 63 to 154 ) and an a-helix (H4; residues 193 to 204) that packs against one of the $\beta$-sheets through hydrophobic interactions [13]. The pVHL-Elongin $C$ complex nucleates a complex containing Elongin B, CUL2 and RBX1, forming the VCB-CR complex (Figure 4), which is thus resistant to proteasomal degradation through their interactions with each other [8]. The a-domain has an important role in the maintenance of the spatial conformation stability of pVHL [14]; the $\beta$-domain binds directly to HIF-a (HIF-1a or HIF-2a) and participates in the degradation of the HIF subunit under aerobic conditions. Previous data shows that the HIFa peptide binds exclusively to the $\beta$-domain of pVHL [15]. A six-residue NH2-terminal segment (residues 561 to 566 ) that is centered on Hyp ${ }^{564}$ (Figure 4 in blue), a three-letter code for hydroxyproline, is central to the binding of HIF-1a to pVHL $\beta$-domain [9]. The pVHL residues that interact with Hyp ${ }^{564}$, including W117 which is spatially close to Hyp ${ }^{564}$ (3.5A , Figure 4), are highly conserved. And W117R missense mutation of pVHL has been shown to abolish HIF-1a binding [16].

Considering that the mutation c.351G >A introduced a premature stop codon which results in the replacement of tryptophane (TGG) with a stop codon (TGA) at codon 117 (p.Trp117Ter), either it can lead to the production of a truncated protein missing $45 \%$ of its residues including the predicted downstream a-domain (residues 155-192) and the a-helix (H4; residues 193 to 204) part of $\beta$-domain, failling to bind to Elongin C, Elongin B, CUL2 and RBX1 to form the VCB-CR complex, or the protein may be entirely absent due to the Nonsense-Mediated mRNA Decay (NMD), a process that typically degrades transcripts containing premature termination codons (PTCs) in order to prevent translation of unnecessary or aberrant transcripts. According to the ClinGen Haploinsufficiency Score and the prediction of the aforementioned bioinformatics tools, it is likely that aberrant VHL transcripts with the nonsense mutation p.Trp117Ter undergo NMD, thus no protein will be synthesized from the mutant allele. The haploinsufficiency of $V H L$ expression will lead to the loss of function (LoF) of the PVHL, then the accumulation of HIFa and subsequent overexpression of HIF target genes, including VEGF, PDGF $\beta$, TGF a, CyclinD1 and EPO, which play a key role in the process of tumorigenesis, and consequently, results in VHL-associated tumors [17].

Two different nonsense mutations of residue 117 have previously been reported and enrolled in Human Gene Mutation Database (HGMD) (Table 3): a somatic c.350G>A (p.Trp117Ter) mutation was detected in a 64 years female sporadic RCC patient [18], while a somatic c.351G>A (p.Trp117Ter) mutation was found in cell lines UOK163 derived from tumor tissue from patients with renal cell carcinomas [19], and a germline c.351G>A (p.Trp117Ter) mutation was discovered in a kindred with VHL disease without phaeochromocytoma phenotype [20].

Taken together, this variant is classified as pathogenic and the supporting evidence for the pathogenicity of $V H L$ c.351G $>A$ according to the American College of Medical Genetics and Genomics (ACMG)(2015) was as follows: (1) PVS1 (very strong pathogenicity 1): variation may lead to loss of gene function; (2) PS1 (strong pathogenicity 1): known disease mutation at this position enrolled in HGMD [18-20]; (3) PM2 (moderate pathogenicity 2): absent from public databases; (4) PP1 (supporting pathogenicity 1): cosegregation with disease in all affected family members in the $V H L$ gene that was definitely known to cause the VHL disease; (5) PP3 (supporting pathogenicity 3): PROVEAN, Gerp++, PhyloP, PhastCons, Polyphen-2, MutationTaster, CADD and ClinGen Haploinsufficiency Score predicted that the variant affects the gene products and is harmful in the conservativeness and structure of the protein. (6) PP4 (supporting pathogenicity 4): patient's phenotype and the family history is highly specific for the VHL disease with a single genetic etiology. 
All of the diagnosed patients examined in this study were classified as type $1 \mathrm{VHL}$, in accordance with the fact that missense mutations are associated with the development of type $2 \mathrm{VHL}$ disease, whereas deletions or mutations that lead to truncation of the VHL protein ( $\mathrm{pVHL}$ ) are primarily associated with the development of type $1 \mathrm{VHL}$ disease [21]. In addition, previous studies have indicated that $V H L$ deletions and protein truncating mutations appear to confer a higher risk of CNS HGBs than missense mutations [20,22,23]. In line with this observation, individuals in the family examined in our study all presented with CNS HGBs, but no manifestation of phaeochromocytoma. At least 4 out of 8 (50\%) of our patients developed retinal angiomas

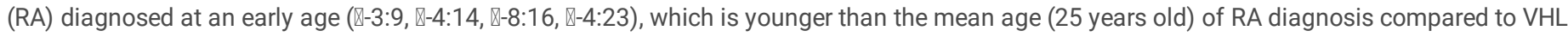
patients in general [21]. This frequency is much higher than those of retinal lesions in VHL patients with intragenic mutations and partial deletions, suggesting that nonsense c.351G>A mutation may confer to a high risk of early onset of RA, which is in contrast to Maher's observation that the risk of RA is slightly higher (45\% vs 37\%) in the missense mutation group than in the deletion/protein truncation group [20]. With regard to the other manifestations, a single pancreatic cyst was detected in the proband, while RC or RCC was diagnosed in the proband's father, aunt and grandfather, suggesting a relatively lower incidence of visceral organs lesion.

This is the first elaborately studied VHL family caused by p.Trp117Ter mutation. Further functional evidence research remains to be conducted to reveal the pathogenesis of $\mathrm{p}$.Trp117Ter.

\section{Conclusions}

We conclude that the p.Trp117Ter nonsense mutation is the causal mutation of this kindred that may lead to clear familial aggregation of VHL disease because of the dysfunction of truncated pVHL via NMD mechanism, and this nonsense mutation is likely to be associated with a higher risk of CNS and retinal HGBs, but a lower risk of visceral organs lesion. Every members of a VHL family with the p.Trp117Ter nonsense mutation should be systematically and comprehensively examined considering the high penetrance (90\%) among mutation carriers, especially in the CNS and retina, and regular follow-up should be strictly conducted to ensure that VHL complications are recognized at a curable stage.

\section{Abbreviations}

VHL, Von Hippel-Lindau; CNS, central nervous system; AD, autosomal dominant; HGB, hemangioblastoma; RA, retinal angiomas; ccRCC, clear-cell renal cell carcinoma; RC, renal cyst; PCC, phaeochromocytoma; PCT, pancreatic cyst and tumor; HIFa, hypoxia-inducible factor-a; PCR, polymerase chain reaction; NMD, Nonsense-Mediated mRNA Decay; HGMD, Human Gene Mutation Database; ACMG, American College of Medical Genetics and Genomics; LoF, loss of function.

\section{Declarations}

\section{Ethics approval and consent to participate}

The study was approved by the Institutional Review Board of Beijing Ditan Hospital.

\section{Consent for publication}

All consent for publication from persons in this study is available on request.

\section{Availability of data and materials}

All sequencing data analysed during the current study is available from the corresponding author on reasonable request.

\section{Competing interests}

The authors declare that they have no competing interests.

\section{Funding}

This research received no specific grant from any funding agency, commercial or not for profit sectors.

\section{Authors' contributions}

DX, LT, FE and WT conceived and designed the research and DX was a major contributor in writing the manuscript. LT and FE handled funding and supervision.

LB, WF, WS, CY, SJ, ZX and WX collected individuals' clinical and imaging data.

LB, LP, CS and LJ analyzed and interpreted the patient clinical and imaging data. All authors read and approved the final manuscript.

\section{Acknowledgements}

We thank the family who participated in this research. 


\section{References}

1. Maher ER, Neumann HP, Richard S: von Hippel-Lindau disease: a clinical and scientific review.Eur J Hum Genet 2011, 19:617-623.

2. Weil RJ, Lonser RR, DeVroom HL, Wanebo JE, Oldfield EH: Surgical management of brainstem hemangioblastomas in patients with von HippelLindau disease.J Neurosurg 2003, 98:95-105.

3. Lonser RR, Butman JA, Huntoon K, Asthagiri AR, Wu T, Bakhtian KD, Chew EY, Zhuang Z, Linehan WM, Oldfield EH: Prospective natural history study of central nervous system hemangioblastomas in von Hippel-Lindau disease.J Neurosurg 2014, 120:1055-1062.

4. Dollfus H, Massin P, Taupin P, Nemeth C, Amara S, Giraud S, Beroud C, Dureau P, Gaudric A, Landais P, Richard S: Retinal hemangioblastoma in von Hippel-Lindau disease: a clinical and molecular study.Invest Ophthalmol Vis Sci 2002, 43:3067-3074.

5. Varshney N, Kebede AA, Owusu-Dapaah H, Lather J, Kaushik M, Bhullar JS: A Review of Von Hippel-Lindau Syndrome.J Kidney Cancer VHL 2017, 4:20-29.

6. Maher ER, Yates JR, Harries R, Benjamin C, Harris R, Moore AT, Ferguson-Smith MA: Clinical features and natural history of von Hippel-Lindau disease.Q J Med 1990, 77:1151-1163.

7. Nordstrom-O'Brien M, van der Luijt RB, van Rooijen E, van den Ouweland AM, Majoor-Krakauer DF, Lolkema MP, van Brussel A, Voest EE, Giles RH: Genetic analysis of von Hippel-Lindau disease. Hum Mutat 2010, 31:521-537.

8. Gossage L, Eisen T, Maher ER: VHL, the story of a tumour suppressor gene.Nat Rev Cancer 2015, 15:55-64.

9. JH M, H Y, M I, F G, WG K, NP P: Structure of an HIF-1alpha -pVHL complex: hydroxyproline recognition in signaling.Science (New York, NY) 2002, 296:1886-1889.

10. Stolle C, Glenn G, Zbar B, Humphrey JS, Choyke P, Walther M, Pack S, Hurley K, Andrey C, Klausner R, Linehan WM: Improved detection of germline mutations in the von Hippel-Lindau disease tumor suppressor gene.Hum Mutat 1998, 12:417-423.

11. Peng S, Shepard M, Wang J, Li T, Ning X, Cai L, Zhuang Z, Gong K: Genotype-phenotype correlations in Chinese von Hippel-Lindau disease patients. Oncotarget 2017, 8:38456-38465.

12. Melmon K, Rosen S: Lindau's disease. Review of the literature and study of a large kindred.Am J Med 1964:595-617.

13. Stebbins CE, Kaelin WG, Jr., Pavletich NP: Structure of the VHL-ElonginC-ElonginB complex: implications for VHL tumor suppressor function.Science 1999, 284:455-461.

14. He ZW, Xia L, Deng ZY, Lian AJ, Hu ZM, Li B: Identification of a VHL gene mutation in a Chinese family with Von Hippel-Lindau syndrome.Molecular Medicine Reports 2018, 18:435-440.

15. Ohh M, Park CW, Ivan N, Hoffman MA, Kim TY, Huang LE, Pavletich N, Chau V, Kaelin WG: Ubiquitination of hypoxia-inducible factor requires direct binding to the beta-domain of the von Hippel-Lindau protein.Nature Cell Biology 2000, 2:423-427.

16. Ohh M, Park CW, Ivan M, Hoffman MA, Kim TY, Huang LE, Pavletich N, Chau V, Kaelin WG: Ubiquitination of hypoxia-inducible factor requires direct binding to the beta-domain of the von Hippel-Lindau protein.Nat Cell Biol 2000, 2:423-427.

17. Kaelin WG, Jr.: Molecular basis of the VHL hereditary cancer syndrome.Nat Rev Cancer 2002, 2:673-682.

18. Bailly M, Bain C, Favrot MC, Ozturk M: Somatic mutations of von Hippel-Lindau (VHL) tumor-suppressor gene in European kidney cancers. Int $J$ Cancer 1995, 63:660-664.

19. Gnarra JR, Tory K, Weng Y, Schmidt L, Wei MH, Li H, Latif F, Liu S, Chen F, Duh FM, et al.: Mutations of the VHL tumour suppressor gene in renal carcinoma.Nat Genet 1994, 7:85-90.

20. Maher ER, Webster AR, Richards FM, Green JS, Crossey PA, Payne SJ, Moore AT: Phenotypic expression in von Hippel-Lindau disease: correlations with germline VHL gene mutations.J Med Genet 1996, 33:328-332.

21. Barry RE, Krek W: The von Hippel-Lindau tumour suppressor: a multi-faceted inhibitor of tumourigenesis. Trends in Molecular Medicine 2004, 10:466472.

22. Cybulski C, Krzystolik K, Murgia A, Gorski B, Debniak T, Jakubowska A, Martella M, Kurzawski G, Prost M, Kojder I, et al: Germline mutations in the von Hippel-Lindau (VHL) gene in patients from Poland: disease presentation in patients with deletions of the entire VHL gene.J Med Genet 2002, 39:E38.

23. Franke G, Bausch B, Hoffmann MM, Cybulla M, Wilhelm C, Kohlhase J, Scherer G, Neumann HP: Alu-Alu recombination underlies the vast majority of large VHL germline deletions: Molecular characterization and genotype-phenotype correlations in VHL patients. Hum Mutat 2009, 30:776-786.

\section{Tables}




\begin{tabular}{|c|c|c|}
\hline Gene name & Sequence of primers & Product Size (bp) \\
\hline VHL exon 1 & $\begin{array}{l}\text { 5'- GCGTTCCATCCTCTACCGA -3' } \\
\text { 5'- TTCAGACCGTGCTATCGTCC -3' }\end{array}$ & 523 \\
\hline VHL exon 2 & 5' - GTGGCTCTTTAACAACCTTTGC -3'5'- CCTGTACTTACCACAACAACCTTATC -3' & 208 \\
\hline VHL exon 3 & 5' - GCAAAGCCTCTTGTTCGTTC -3’5'- CAAAAATGCCACCACCTTCT -3' & 504 \\
\hline
\end{tabular}

\begin{tabular}{|c|c|c|c|c|c|c|c|c|c|c|}
\hline & \multirow{2}{*}{$\begin{array}{l}\text { Age at diagnosis or last } \\
\text { evaluation }\end{array}$} & \multirow[t]{2}{*}{ Sex } & \multirow[t]{2}{*}{ Hypertension } & \multirow{2}{*}{$\begin{array}{l}\text { VHL gene mutation } \\
\text { status }\end{array}$} & \multirow{2}{*}{$\begin{array}{l}\text { Clinical } \\
\text { type }\end{array}$} & \multicolumn{5}{|c|}{ Phenotype } \\
\hline & & & & & & $\begin{array}{l}\text { CNS } \\
\text { HGB }\end{array}$ & RA & $\mathrm{RCC} / \mathrm{RC}$ & PCT & PCC \\
\hline $\begin{array}{l}\text { I- } \\
1^{a}\end{array}$ & 60 & $M$ & $Y$ & NA & Type $₫$ & $++^{\mathrm{b}}$ & NA & NA & NA & NA \\
\hline II-1 & 20 & $M$ & $Y$ & NA & Type $₫$ & + & - & + & - & - \\
\hline II-5 & 60 & $\mathrm{~F}$ & $Y$ & NA & Type $₫$ & + & - & - & - & - \\
\hline $\begin{array}{l}\text { III- } \\
1\end{array}$ & 49 & $\mathrm{~F}$ & $Y$ & $Y$ & 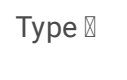 & + & - & + & - & - \\
\hline $\begin{array}{l}\text { III- } \\
3\end{array}$ & 9 & $M$ & $Y$ & $Y$ & Type $\rrbracket$ & + & + & + & - & - \\
\hline $\begin{array}{l}\text { III- } \\
8\end{array}$ & 16 & $M$ & $\mathrm{~N}$ & $Y$ & Type $\rrbracket$ & + & + & - & - & - \\
\hline$\bigotimes-1$ & 25 & $\mathrm{~F}$ & $\mathrm{~N}$ & $Y$ & ND & - & - & - & - & - \\
\hline$\bigotimes-2$ & 13 & $M$ & $\mathrm{~N}$ & $Y$ & 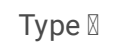 & + & - & - & - & - \\
\hline$\bigotimes-3$ & 23 & $M$ & $\mathrm{~N}$ & $Y$ & Type $\rrbracket$ & + & + & - & + & - \\
\hline$\bigotimes-4$ & 14 & $\mathrm{~F}$ & $\mathrm{~N}$ & $Y$ & Type $\rrbracket$ & + & + & - & - & - \\
\hline \multicolumn{11}{|c|}{$\begin{array}{l}\text { Abbreviations: VHL, von Hippel-Lindau; M, man; F, female; Y, yes; N, no; NA, not available; ND, not diagnosed; CNS, central nervous system; HGB, } \\
\text { hemangioblastoma; RA, retinal angioma; RCC, renal cell carcinoma; RC, renal cysts; PCT, pancreatic cysts or tumors; PCC, phaeochromocytoma; EC, } \\
\text { epididymal/ovarian cystadenoma. }\end{array}$} \\
\hline \multicolumn{11}{|c|}{ a Death before diagnosis. } \\
\hline \multicolumn{11}{|c|}{ b diagnosis not confirmed. } \\
\hline
\end{tabular}

\begin{tabular}{|c|c|c|c|c|c|c|c|c|c|}
\hline $\begin{array}{l}\text { Public } \\
\text { database }\end{array}$ & $1000 \mathrm{G}$ & ESP6500 & ExAC & gnomAD & $\mathrm{db}$ & & & & \\
\hline $\begin{array}{l}\text { Allele } \\
\text { frequency }\end{array}$ & 0 & 0 & 0 & 0 & 0 & & & & \\
\hline $\begin{array}{l}\text { Bioinformatics } \\
\text { tool }\end{array}$ & PROVEAN & Gerp++ & PhyloP & PhastCons & $\begin{array}{l}\text { Polyphen- } \\
2\end{array}$ & MutationTaster & CADD & Haploinsufficiency & $\mathrm{pLI}$ \\
\hline Prediction & $-16.57 ; D^{a}$ & 5.07 & 4.071 & 1 & $1 ; D^{b}$ & $1 ; D^{C}$ & 6.051 & 3 & 0.034 \\
\hline
\end{tabular}




\begin{tabular}{|lllll|}
\hline \multicolumn{4}{|l}{ Table 4. Published reports of VHL patients with nonsense mutation } & VHL p.Trp117Ter described in the Human Gene Mutation Database (HGMD) \\
Reference & Nucleotide change (c.DNA) & Mutation type & Phenotype & Gender/Age \\
\hline Bailly M [12] & c.350G $>$ A & Somatic & RCC & F/64 \\
\hline Gnarra JR [13] & c.351G>A & Somatic & RCC & Cell lines UOK163 \\
\hline Maher ER [14] & c.351G $>$ A & Germline & VHL & NA \\
\hline
\end{tabular}

\section{Figures}

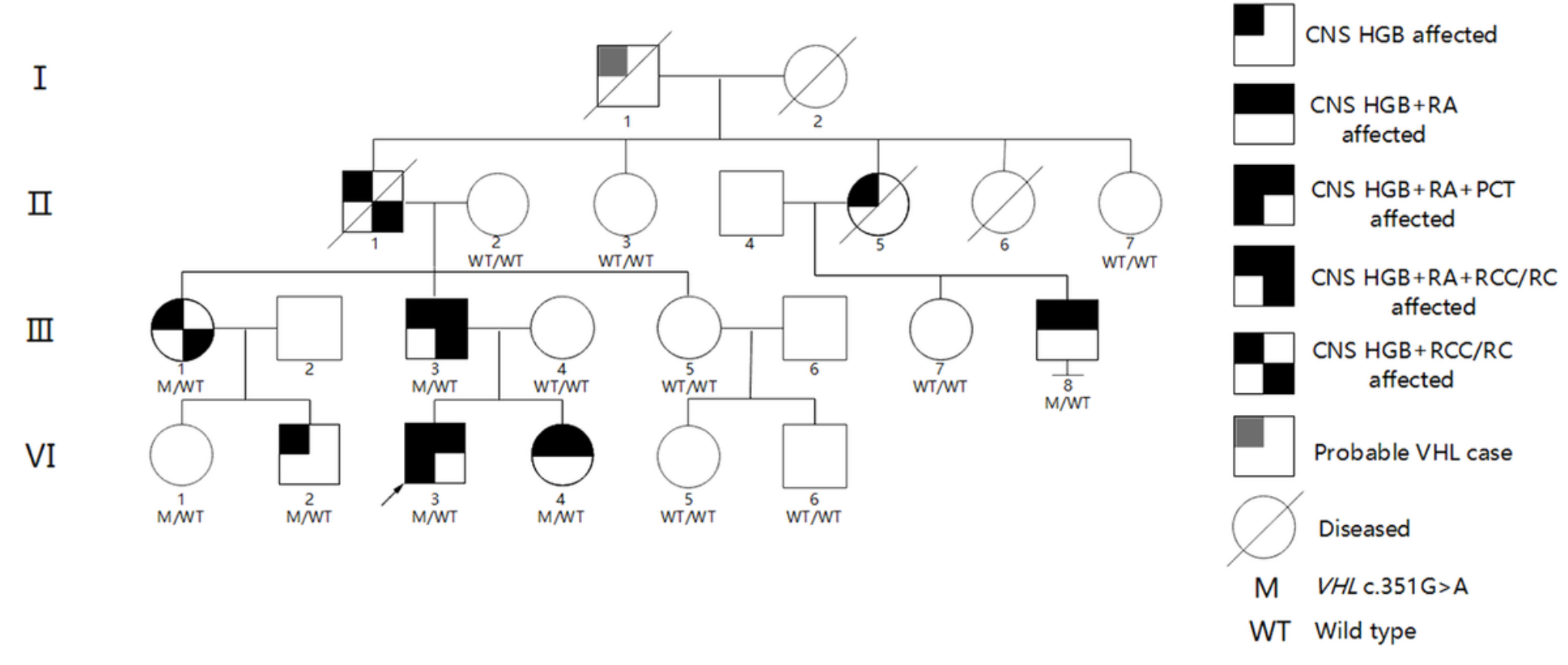

Figure 1

Pedigree and genotype segregation in the family with VHL disease. Squares indicate males; circles represent females; arrow indicates the proband. WT/WT represents both wild type alleles, i.e. bi-allelic, while M/WT designates VHL c.351G>A pathogenic variant as heterozygous allele.
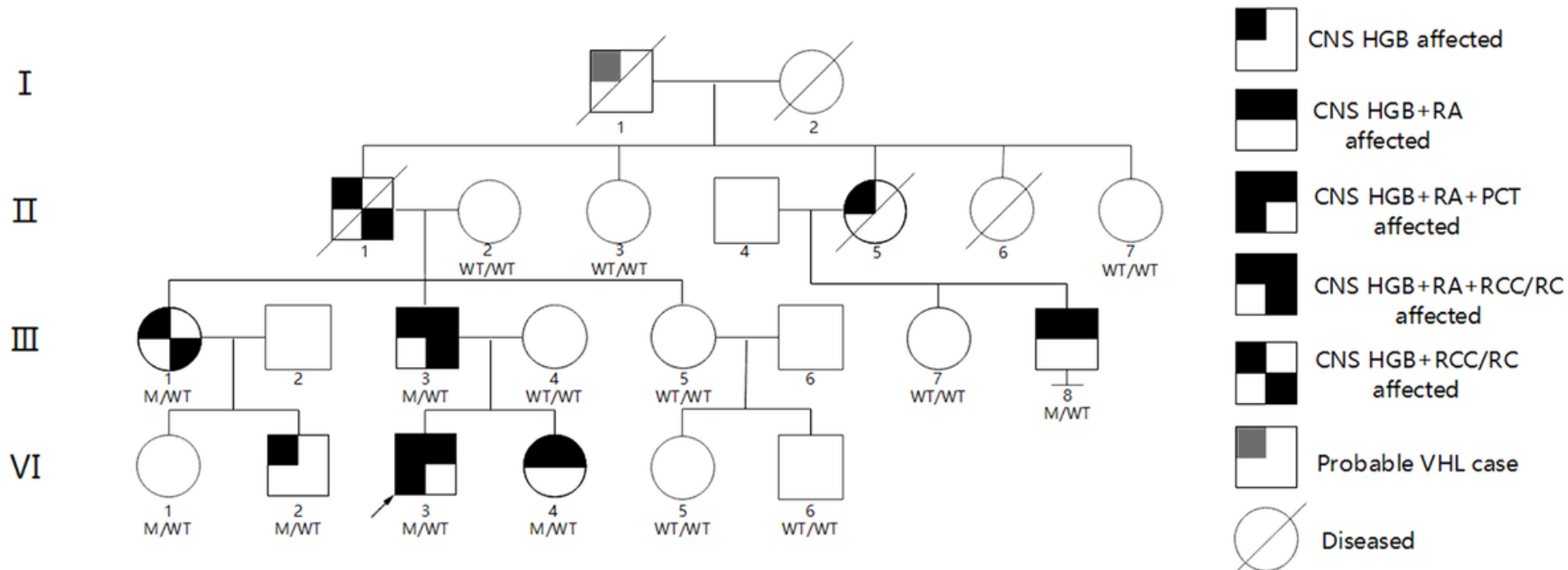

Probable VHL case

Diseased

M VHL c.351G >A

WT Wild type

Figure 1

Pedigree and genotype segregation in the family with VHL disease. Squares indicate males; circles represent females; arrow indicates the proband. WT/WT represents both wild type alleles, i.e. bi-allelic, while M/WT designates VHL c.351G>A pathogenic variant as heterozygous allele. 


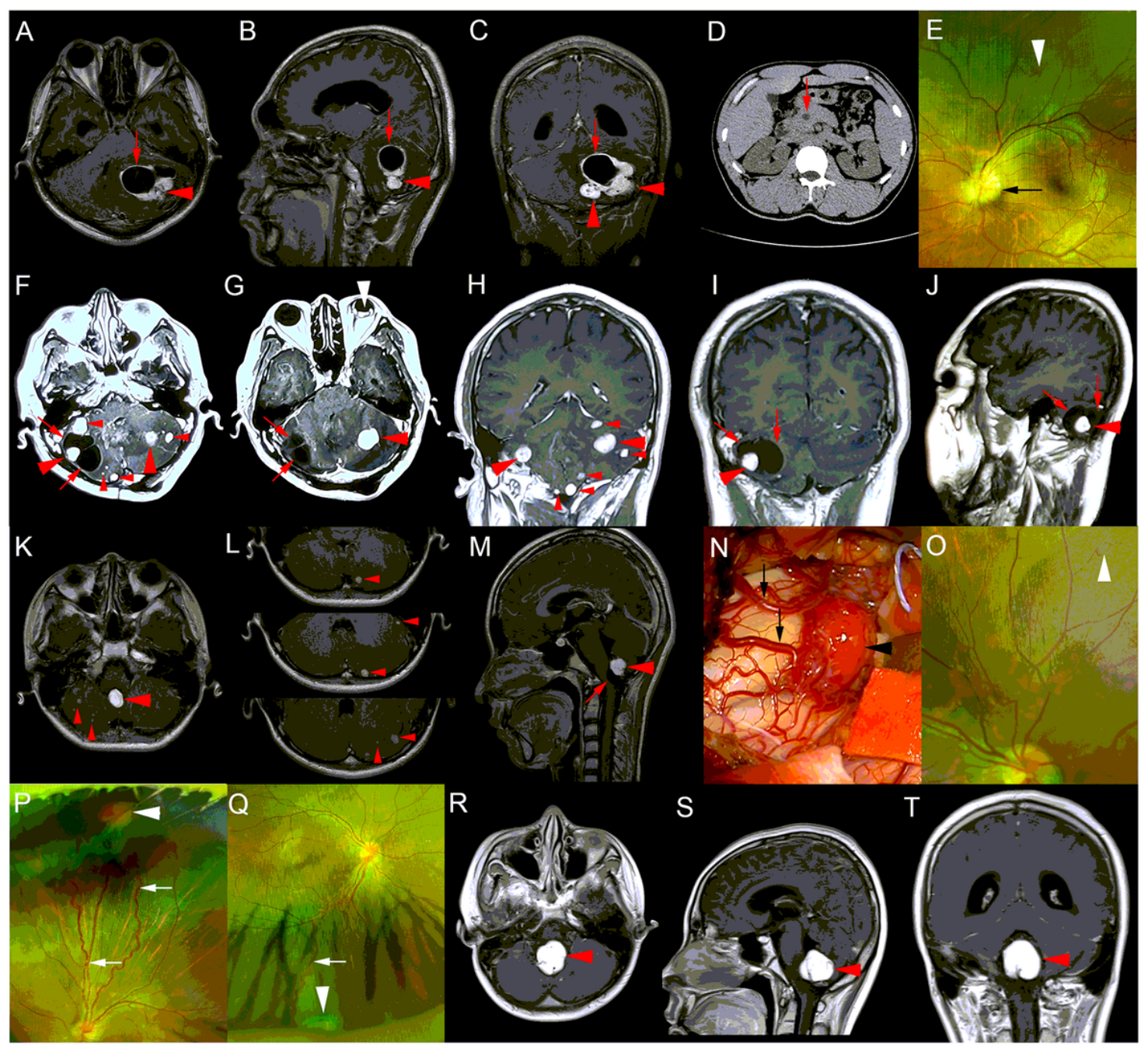

Figure 2

Imaging manifestations of VHL patients in this family. (A-C) Axial, sagittal and coronal T1-weighted contrast-enhanced MRI reveals an enhancing mural nodule (red arrowhead) with a large peritumoral cyst (red arrow) in the left cerebellar hemisphere exerting a mass effect upon the midline and fourth ventricle. (D) Abdominal computed tomography scan reveals a cyst (red arrow) in the pancreatic head. (E) Fundoscopic view of an atypical angiomas (white arrowhead) on the left eye upper retina and the optic disc presents edematous (black arrow). (F-J) Axial, coronal and sagittal T1-weighted contrast-enhanced MRI reveals an enhancing mural nodule (big red arrowhead) with two large accompanying cysts (red arrow) in the right cerebellar hemisphere and several isolated enhancing hemangioblastomas (little red arrowhead). (G) Axial T1-weighted contrast-enhanced MRI detects the left eye retinal hemangioblastoma (white arrowhead). (K-M) Axial and sagittal T1-weighted contrast-enhanced MRI shows multiple enhancing lesions (red arrowhead) widely distributed in the cerebellum with the biggest one (big red arrowhead) near the brainstem with an accompanying cyst (red arrow). ( $\mathrm{N}$ ) Intraoperative image of the brainstem-nearby hemangioblastoma nidus (black arrowhead) and its feeding artery (black arrows), posterior inferior cerebellar artery (PICA). (O-Q) Fundoscopic view of a angiomas (white arrowhead) on the right eye upper retina, and of retinal hemangioblastomas on both eyes at the upper and lower poles of retina respectively, which have easily recognizable globular reddish appearance (white arrowhead) with dilated feeding arteries (white arrows). (R-T) Axial, sagittal and coronal T1-weighted contrast-enhanced MRI reveal a large enhancing hemangioblastoma (red arrowhead) located deep in the cerebellum near the brainstem. 


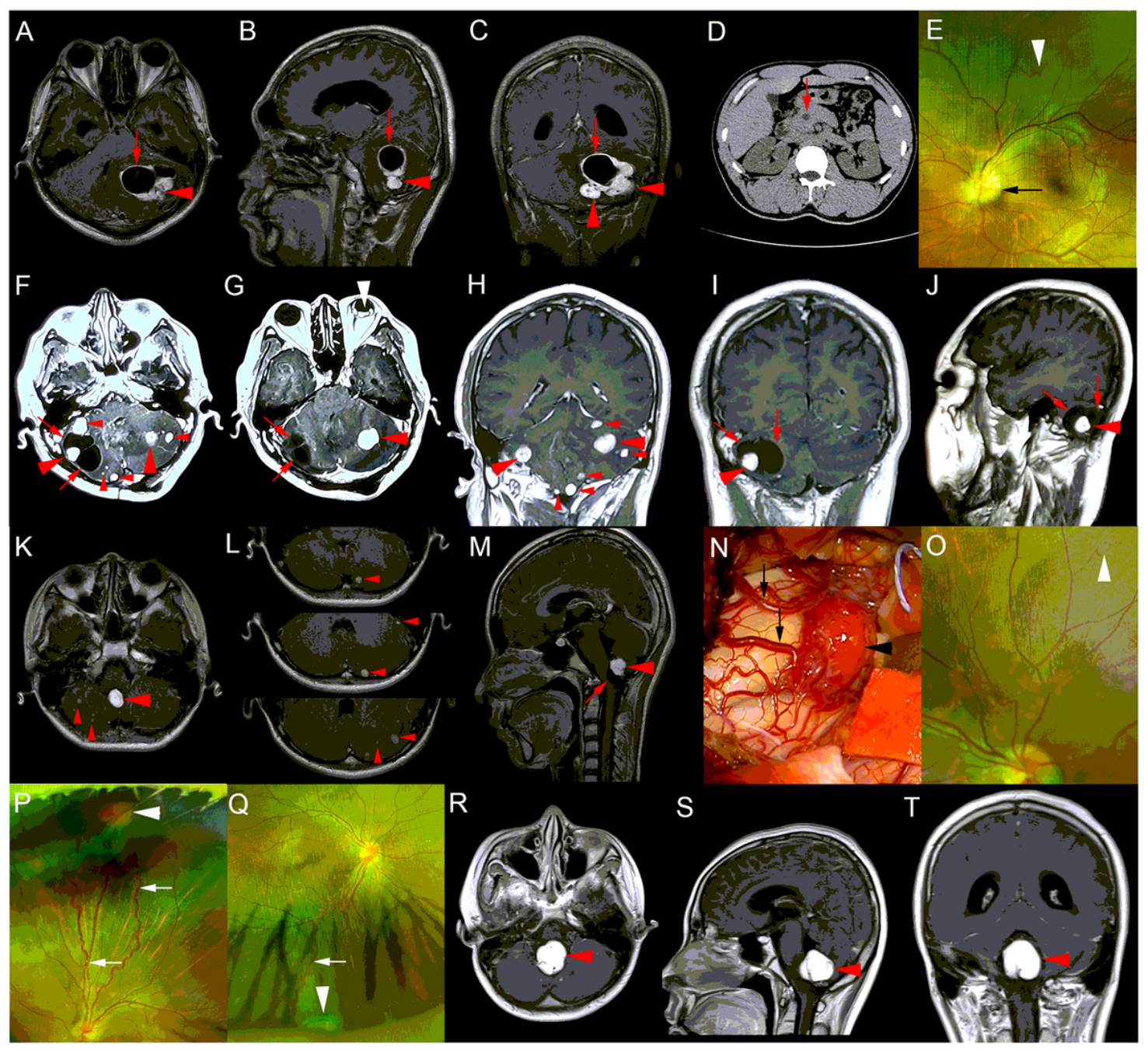

Figure 2

Imaging manifestations of VHL patients in this family. (A-C) Axial, sagittal and coronal T1-weighted contrast-enhanced MRI reveals an enhancing mural nodule (red arrowhead) with a large peritumoral cyst (red arrow) in the left cerebellar hemisphere exerting a mass effect upon the midline and fourth ventricle. (D) Abdominal computed tomography scan reveals a cyst (red arrow) in the pancreatic head. (E) Fundoscopic view of an atypical angiomas (white arrowhead) on the left eye upper retina and the optic disc presents edematous (black arrow). (F-J) Axial, coronal and sagittal T1-weighted contrast-enhanced MRI reveals an enhancing mural nodule (big red arrowhead) with two large accompanying cysts (red arrow) in the right cerebellar hemisphere and several isolated enhancing hemangioblastomas (little red arrowhead). (G) Axial T1-weighted contrast-enhanced MRI detects the left eye retinal hemangioblastoma (white arrowhead). (K-M) Axial and sagittal T1-weighted contrast-enhanced MRI shows multiple enhancing lesions (red arrowhead) widely distributed in the cerebellum with the biggest one (big red arrowhead) near the brainstem with an accompanying cyst (red arrow). ( $\mathrm{N}$ ) Intraoperative image of the brainstem-nearby hemangioblastoma nidus (black arrowhead) and its feeding artery (black arrows), posterior inferior cerebellar artery (PICA). (O-Q) Fundoscopic view of a angiomas (white arrowhead) on the right eye upper retina, and of retinal hemangioblastomas on both eyes at the upper and lower poles of retina respectively, which have easily recognizable globular reddish appearance (white arrowhead) with dilated feeding arteries (white arrows). (R-T) Axial, sagittal and coronal T1-weighted contrast-enhanced MRI reveal a large enhancing hemangioblastoma (red arrowhead) located deep in the cerebellum near the brainstem. 


\section{A}
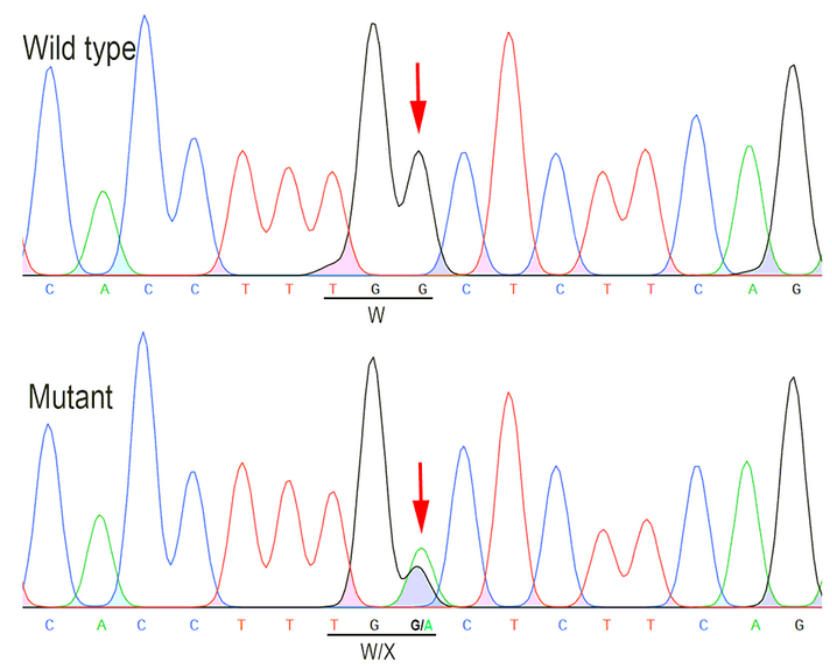

B

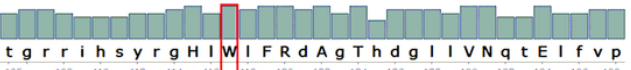

Homo sapiens Canis lupus familiaris Bos taurus Mus musculus Rattus norvegicus Xenopus tropicalis Gallus gallus Macaca mulatta
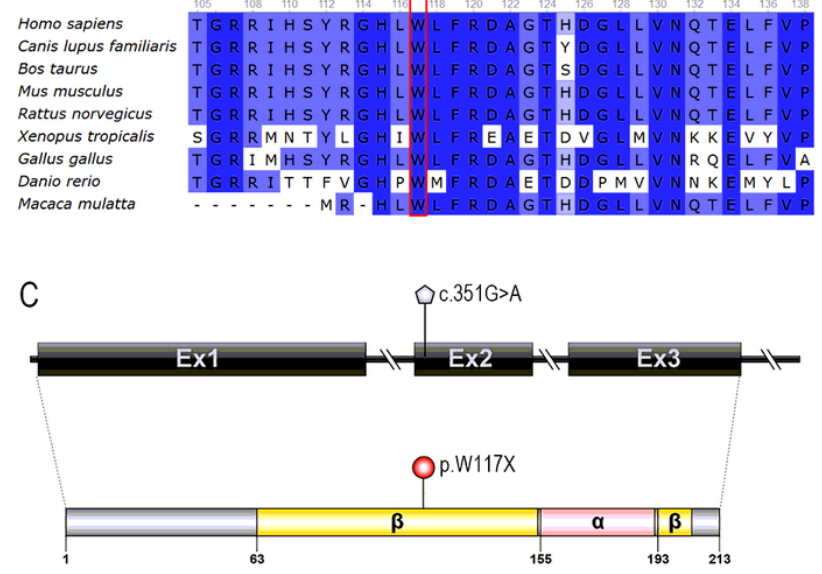

Figure 3

Sequencing detection, evolutionary conservation and location of the VHL c.351G>A variant. (A) Sanger sequencing results of the mutated fragments in normal members (upper panal) and heterozygous mutant patients (lower panal) in the family. (B) Multiple species sequence alignment of pVHL amino acids flanking the nonsense mutated residue highlighted by a red box revealed that the tryptophane at the 117th amino acid site and the downstream residues is evolutionarily conserved. (C) VHL c.351G>A (p.Trp117Ter) variant mapping, located in exon 2 of VHL gene, within the $\beta$-domain of pVHL. 


\section{A}
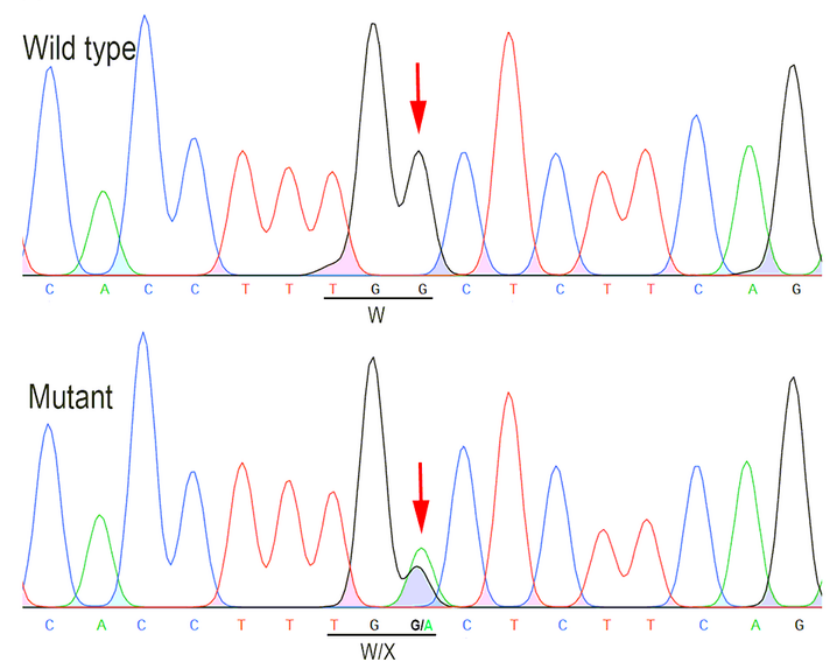

B

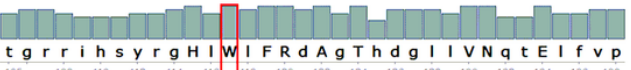

Homo sapiens Canis lupus familiaris Bos taurus Mus musculus Rattus norvegicus Xenopus tropicalis Gallus gallus Macaca mulatta
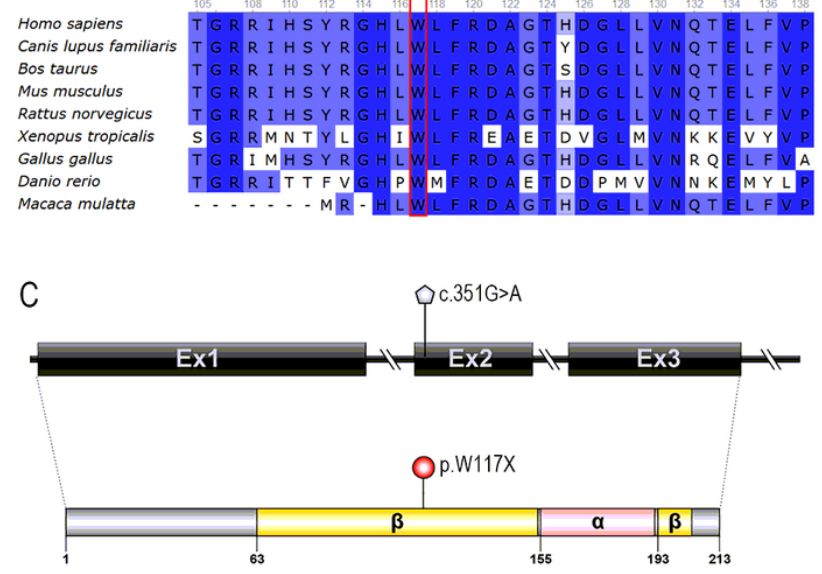

Figure 3

Sequencing detection, evolutionary conservation and location of the VHL c.351G>A variant. (A) Sanger sequencing results of the mutated fragments in normal members (upper panal) and heterozygous mutant patients (lower panal) in the family. (B) Multiple species sequence alignment of pVHL amino acids flanking the nonsense mutated residue highlighted by a red box revealed that the tryptophane at the 117th amino acid site and the downstream residues is evolutionarily conserved. (C) VHL c.351G>A (p.Trp117Ter) variant mapping, located in exon 2 of VHL gene, within the $\beta$-domain of pVHL. 


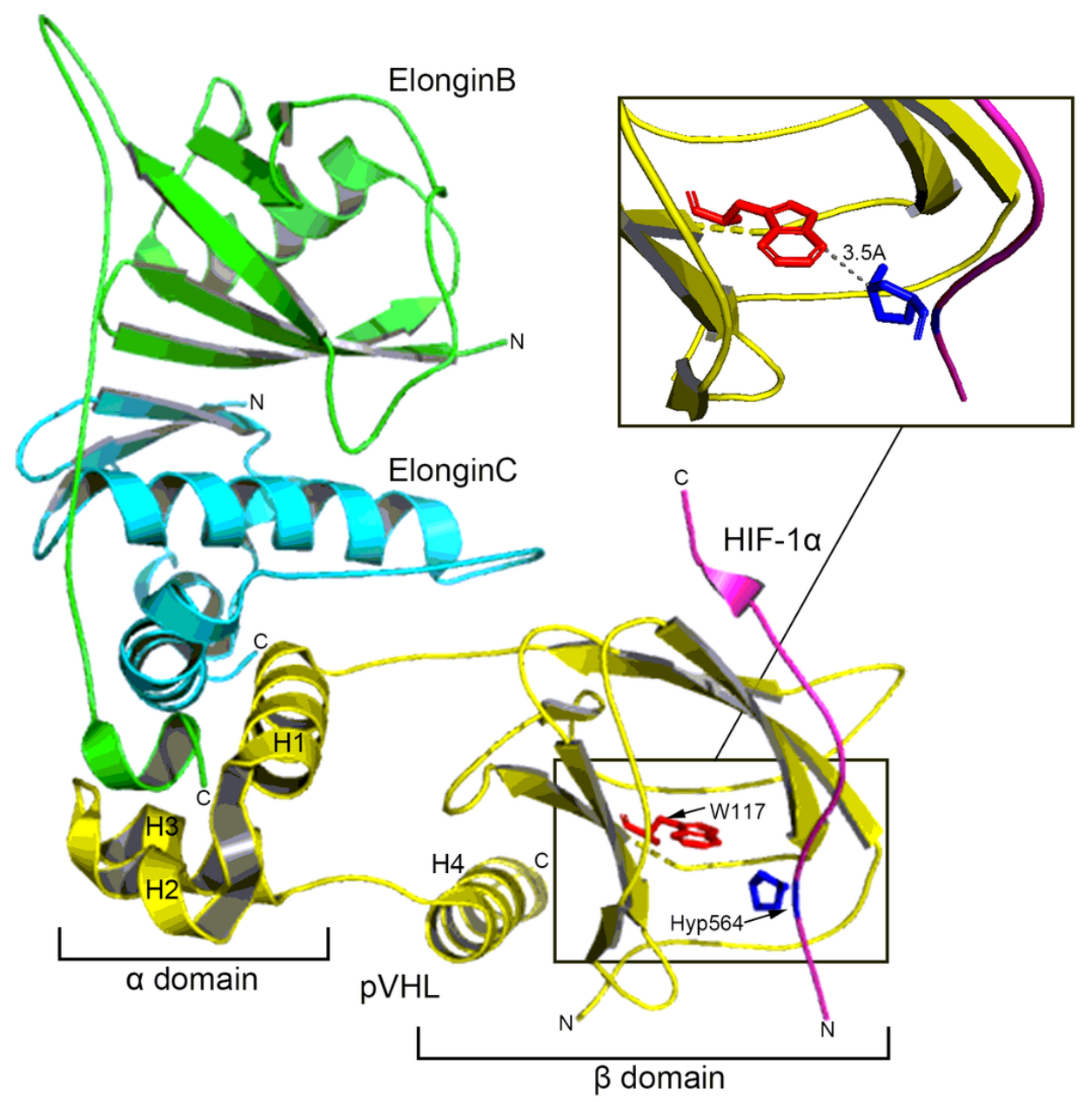

Figure 4

Schematic representation of HIF-1a bound to the $\beta$ domain of pVHL in the pVHL-ElonginB-ElonginC complex in ribbon diagram. $\mathrm{pVHL}$ (yellow) contains two domains, the a-domain and the $\beta$-domain. The a-domain consists of three a-helices $(\mathrm{H} 1, \mathrm{H} 2$ and $\mathrm{H} 3$ ), whereas the $\beta$-domain contains seven-stranded $\beta$ sandwich and an a-helix (H4). pVHL together with elongin C (cyan), elongin B (green), cullin 2 (not shown) and the RING finger protein RBX1(not shown), forms the VCB-CR complex. HIF-1a (pink) binds directly to $\beta$-domain, made by the N segment, in particular by Hyp564 (blue) of the HIF-1a. The mutated amino acid point of W117 (red) is spatially close to Hyp564 (at least 3.5A). 


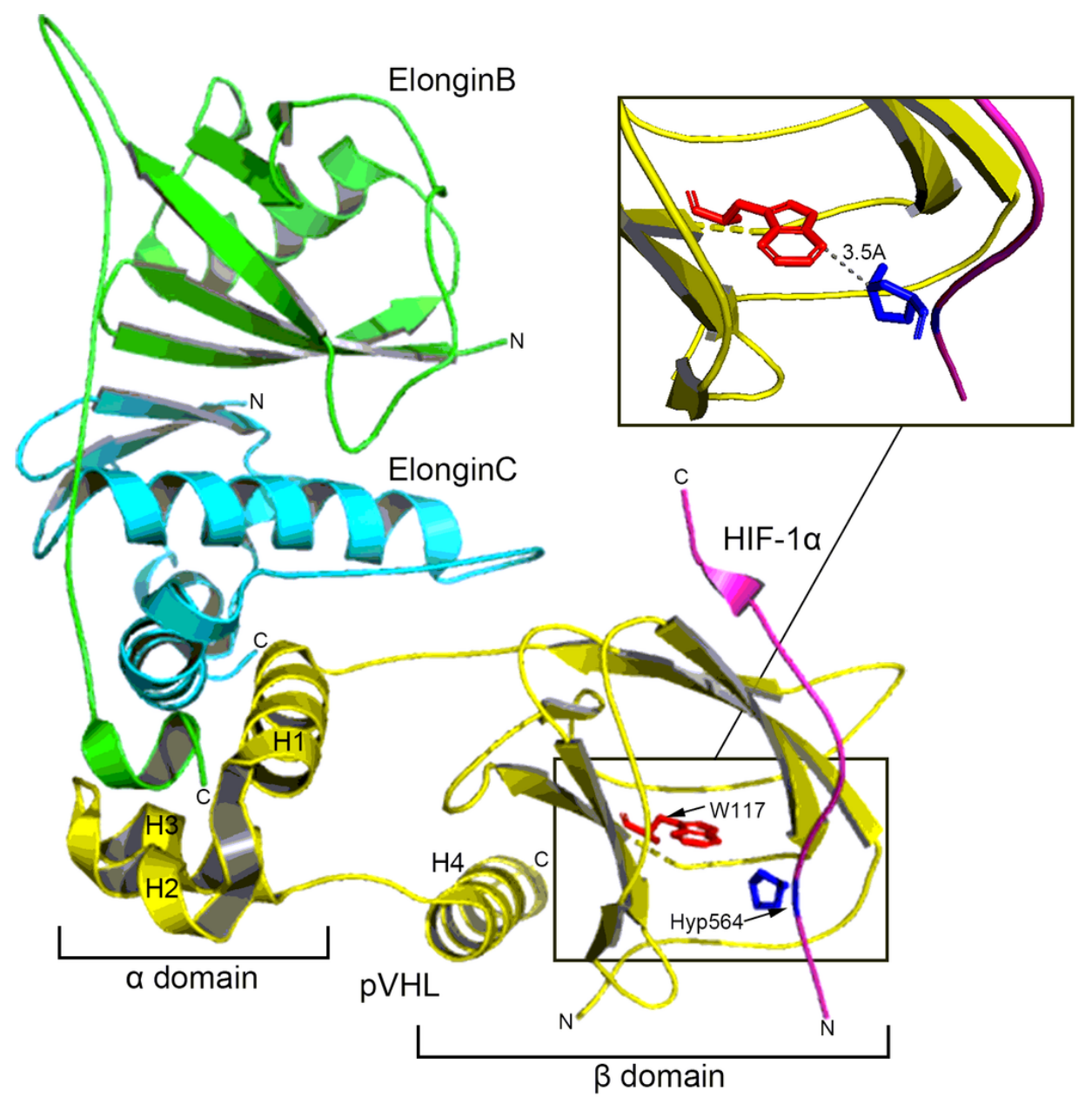

Figure 4

Schematic representation of HIF-1a bound to the $\beta$ domain of pVHL in the pVHL-ElonginB-ElonginC complex in ribbon diagram. $\mathrm{pVHL}$ (yellow) contains two domains, the a-domain and the $\beta$-domain. The a-domain consists of three a-helices $(\mathrm{H} 1, \mathrm{H} 2$ and $\mathrm{H} 3$ ), whereas the $\beta$-domain contains seven-stranded $\beta$ sandwich and an a-helix (H4). pVHL together with elongin C (cyan), elongin B (green), cullin 2 (not shown) and the RING finger protein RBX1(not shown), forms the VCB-CR complex. HIF-1a (pink) binds directly to $\beta$-domain, made by the N segment, in particular by Hyp564 (blue) of the HIF-1a. The mutated amino acid point of W117 (red) is spatially close to Hyp564 (at least 3.5A).

\section{Supplementary Files}

This is a list of supplementary files associated with this preprint. Click to download.

- Figures1.tif

- FigureS1.tif 